\title{
Adaptive Modulation Techniques for Duplex OFDM Transmission
}

\author{
Thomas Keller and Lajos Hanzo
}

\begin{abstract}
The design tradeoffs of turbo-coded burst-by-burst adaptive orthogonal frequency division multiplex (OFDM) wideband transceivers are analyzed. We demonstrate that upon aiming for a higher throughput a higher proportion of low-quality OFDM subcarriers has to be used for the transmission of inherently vulnerable high-order modem modes, transmitting several bits per subcarrier. Upon invoking turbo coding and adjusting the modem mode switching regime near-error-free performance can be achieved at the cost of a reduced throughput. Various blind modem mode detection techniques have also been investigated and the most complex channel coding trellis-based detection algorithm was found to be the most powerful. Last, the design tradeoffs of spectral pre-equalization have been explored and qualtified. We concluded that AOFDM provides a convenient framework for adjusting the required target integrity and throughput both with and without turbo channel coding.
\end{abstract}

\section{BACKGROUND AND MOTIVATION}

$\mathbf{S}$ TEELE and Webb [1], [2] proposed burst-by-burst adaptive quadrature amplitude modulation (AQAM) [2] for exploiting the time-variant Shannonian channel capacity of narrowband fading channels. Their work stimulated further research at the University of Osaka by Sampei et al., investigating variable coding rate concatenated coded schemes [3], at the University of Stanford by Goldsmith et al., studying the effects of variable-rate, variable-power arrangements [4] and at Southampton University in the U.K., researching the associated latency-, netwoking - as well as interference aspects of AQAM [5], [6]. The transmitter's modulation modes employed are reconfigured on a burst-by-burst basis, depending on the instantaneous perceived wireless channel quality. In order to prevent potential error burst due to a low expected instantaneous channel quality the transmitter can also be temporarily disabled, while the data is delayed and buffered, until the channel quality improves, provided that the associated delay is not excessive for the service supported. Hence the distribution of channel errors becomes typically less bursty, than in conjunction with nonadaptive modems, which potentially increases the channel coding gains [7]. Furthermore, the soft-decision channel codec metrics can be also invoked in estimating the instantaneous channel quality [7].

Recently block turbo coded wideband AQAM transceivers have also been proposed for dispersive wideband channels in conjunction with conventional decision feedback equalizers [7], [8] (DFE), where the mean squared error (MSE) at the DFE's

Manuscript received June 7, 1999; revised April 6, 2000. This work was supported in part by the EPSRC, U.K., and in part by the European Commission.

The authors are with the Department of Electronics and Computer Science, University of Southampton, SO17 1BJ, U.K. (e-mail: 1h@ecs.soton.ac.uk).

Publisher Item Identifier S 0018-9545(00)07919-6. output was used as the channel quality metric, controlling the choice of modes modes. An alternative neural-network radial basis function (RBF) DFE-based AQAM modem design was proposed in [9], where the RBF DFE provided the channel quality estimates for the modem mode switching regime.

Further recent work on combining various conventional—rather than iteratively decoded turbo coding—schemes with adaptive modulation has been reported by Matsuoka et al. [3], Lau et al. [10], and Goldsmith et al. [11]. For data transmission systems, which do not necessarily require a low transmission delay, variable - throughput adaptive schemes can be devised, which operate efficiently in conjunction with powerful error correction codecs, such as long block length turbo codes [37]. By contrast, fixed rate burst-by-burst adaptive systems, which sacrifice a guaranteed bit error rate (BER) performance for the sake of maintaining a fixed data throughput, are more amenable to employment in the context of low-delay interactive speech and video communications systems. The above burst-by-burst adaptive principles can also be extended to adaptive orthogonal frequency division multiplexing (AOFDM) schemes [12], and to adaptive joint-detection-based code division multiple access (ACDMA) arrangements [13].

The associated AQAM principles were invoked in the context of parallel AOFDM modems also by Czylwik et al. [24], Fischer [25], and Chow et al. [26]. Adaptive subcarrier selection has been demonstrated by Rohling et al. [28] for BER performance improvements, as well as for crest-factor reduction by Schmidt et al. [29]. Further crest-factor investigations have been conducted by Jones et al.in the U.K. [30], [31]. Multi-user uplink access with adaptive subcarrier allocation has been studied by Grünheid et al. [32]. Without completeness, further significant advances over benign, slowly varying dispersive Gaussian fixed links - rather than over hostile wireless links-are due to Chow et al. [26] from the United States, rendering OFDM the dominant solution for asymmetric digital subscriber loop (ADSL) applications, potentially up to bitrates of $54 \mathrm{Mb} / \mathrm{s}$. In Europe, OFDM has been favored for both digital audio broadcasting (DAB) and digital video broadcasting [17], [18] (DVB) as well as for high-rate wireless asynchronous transfer mode (WATM) systems due to its ability to combat the effects of highly dispersive channels [19]. The notion of adaptive bit allocation in the context of OFDM was proposed as early as 1989 by Kalet [27], which was further developed by Chow et al. [26] and was refined for duplex wireless links, for example in [12]. Last, various OFDM-based speech and video systems were proposed in [15] and [16]. The cochannel interference sensitivity of OFDM can be mitigated with the aid of adaptive beam-forming [22], [23]. 


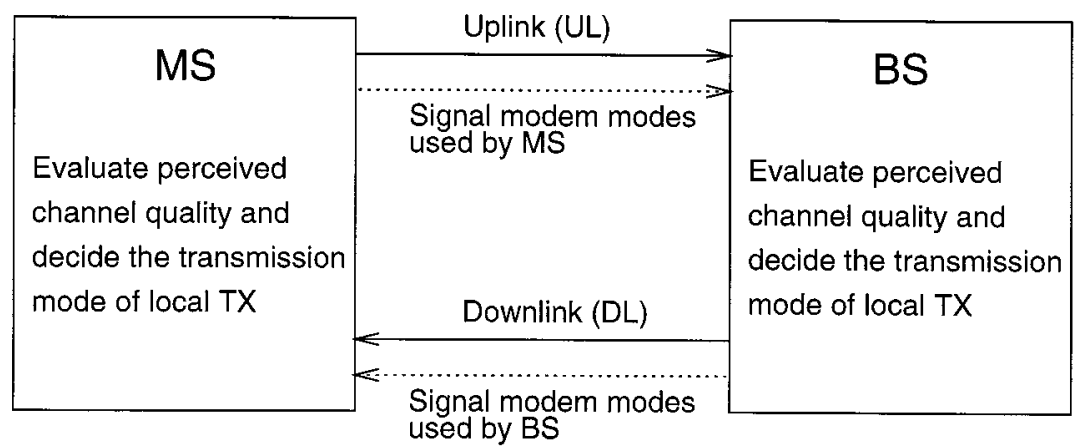

(a)

\begin{tabular}{|c|c|c|}
\hline & Uplink (UL) & \\
\hline $\begin{array}{l}\text { Evaluate perceived } \\
\text { channel quality and } \\
\text { signal the requested } \\
\text { transmission mode }\end{array}$ & $\begin{array}{l}\text { Signal modem modes } \\
\text { to be used by BS } \\
\text { Downlink (DL) }\end{array}$ & $\begin{array}{l}\text { Evaluate perceived } \\
\text { channel quality and } \\
\text { signal the requested } \\
\text { transmission mode }\end{array}$ \\
\hline to ine bs & & to the MS IX \\
\hline
\end{tabular}

(b)

\begin{tabular}{|c|c|c|}
\hline \multirow{8}{*}{$\begin{array}{l}\qquad \text { MS } \\
\text { - Evaluate perceived } \\
\text { channel quality and } \\
\text { decide the transmission } \\
\text { mode of local TX } \\
\text { - Infer the BS TX's } \\
\text { transmission mode } \\
\text { blindly }\end{array}$} & Uplink (UL) & \multirow{8}{*}{$\begin{array}{l}\text { BS } \\
\text { - Evaluate perceived } \\
\text { channel quality and } \\
\text { decide the transmission } \\
\text { mode of local TX } \\
\text { - Infer the BS TX's } \\
\text { transmission mode } \\
\text { blindly }\end{array}$} \\
\hline & & \\
\hline & no signailing & \\
\hline & & \\
\hline & & \\
\hline & & \\
\hline & Downlink (DL) & \\
\hline & no signalling & \\
\hline
\end{tabular}

(c)

Fig. 1. Signaling scenarios in adaptive modems.

AOFDM transmission parameter adaptation is an action of the transmitter in response to time-varying channel conditions. It is only suitable for duplex communication between two stations, since the transmission parameter adaptation relies on some form of channel estimation and signaling. In order to efficiently react to the changes in channel quality, the following steps have to be taken:

- Channel quality estimation: In order to appropriately select the transmission parameters to be employed for the next transmission, a reliable prediction of the channel quality during the next active transmit timeslot is necessary.

- Choice of the appropriate parameters for the next transmission: Based on the prediction of the expected channel conditions during the next timeslot, the transmitter has to select the appropriate modulation schemes for the subcarriers.

- Signaling or blind detection of the employed parameters: The receiver has to be informed, as to which set of demod- ulator parameters to employ for the received packet. This information can either be conveyed within the packet, at the cost of loss of useful data bandwidth, or the receiver can attempt to estimate the parameters employed at the transmitter by means of blind detection mechanisms.

Depending on the channel characteristics, these operations can be performed at either of the duplex stations, as shown in Fig. 1. If the channel is reciprocal, then the channel quality estimation for each link can be extracted from the reverse link, and we refer to this regime as open-loop adaptation. In this case, the transmitter needs to communicate the transmission parameter set to the receiver [Fig. 1(a)], or the receiver can attempt blind detection of the transmission parameters employed [Fig. 1(c)].

If the channel is not reciprocal, then the channel quality estimation has to be performed at the receiver of the link. In this case, the channel quality measure or the set of requested transmission parameters is communicated to the transmitter in the reverse link [Fig. 1(b)]. This mode is referred to as closed-loop adaptation. 


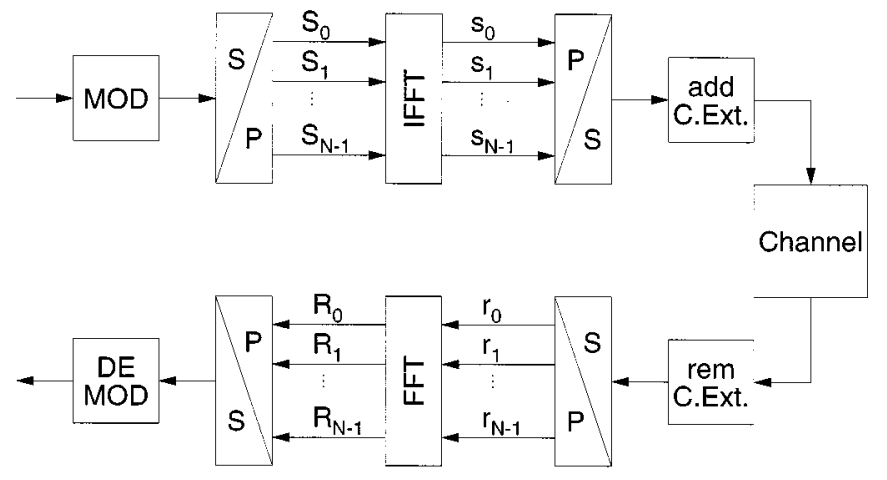

Fig. 2. Schematic model of the OFDM system.

Against this backround in this contribution the design tradeoffs of turbo-coded, pre-equalized fixed- and variable-rate burst-by-burst adaptive OFDM wideband transceivers are analyzed. We will demonstrate that AOFDM provides a convenient framework for adjusting the required target integrity and throughput both with and without turbo channel coding. The remainder of this paper is organized as follows. Section II highlights the philosophy of our AOFDM modem design, invoking various modem mode switching regimes and addressing also a variety of modem mode blind detection algorithms. Section III investigates the effects of spectral predistortion, leading to our discussions in Section IV, comparing the various system design tradeoffs and summarizing our future research efforts. Let us now focus our attention on the various AOFDM system design options.

\section{ADAPTIVE MODULATION FOR OFDM}

\section{A. System Model}

The system model of the $N$-subcarrier OFDM modem is shown in Fig. 2 [33]. At the transmitter, the modulator generates $N$ data symbols $S_{n}, 0 \leq n \leq N-1$, which are multiplexed to the $N$ subcarriers. The time-domain samples $s_{n}$ transmitted during one OFDM symbol are generated by the inverse fast Fourier transform (IFFT) and transmitted over the channel after the cyclic extension (C. Ext.) has been inserted. The channel is modeled by its time-variant impulse response $h(\tau, t)$ and additive white Gaussian noise (AWGN). At the receiver, the cyclic extension is removed from the received time-domain samples, and the data samples $r_{n}$ are fast Fourier transformed (FFT), in order to yield the received frequency-domain data symbols $R_{n}$.

The channel's impulse response is assumed to be constant for the duration of one OFDM symbol, therefore it can be characterized for each OFDM symbol period by the $N$-point Fourier transform of the impulse response, which is referred to as the frequency domain channel transfer function $H_{n}$. The received data symbols $R_{n}$ can be expressed as

$$
R_{n}=S_{n} \cdot H_{n}+n_{n}
$$

where $n_{n}$ is an AWGN sample. Coherent detection is assumed for the system, therefore, the received data symbols $R_{n}$ have

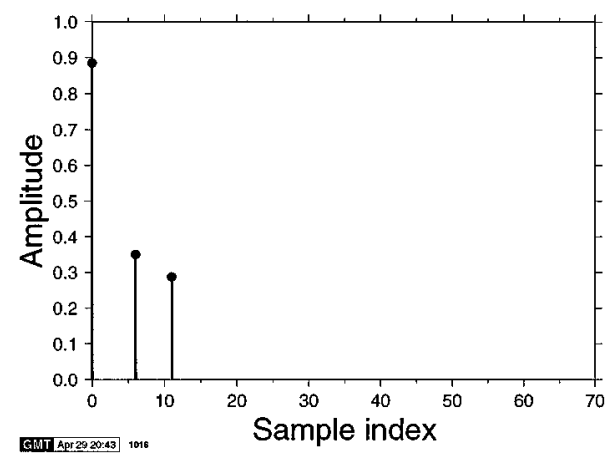

(a)
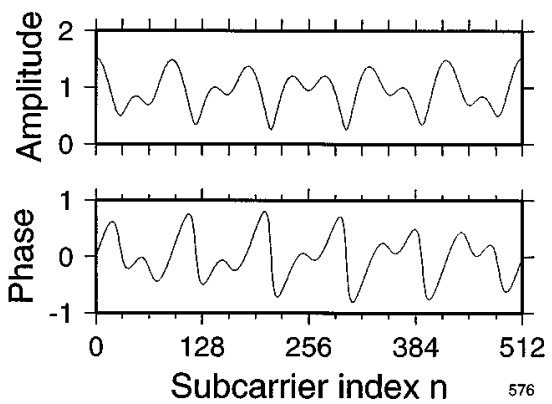

(b)

Fig. 3. Wideband channel: (a) unfaded symbol spaced impulse response and (b) corresponding frequency domain channel transfer function.

to be de-faded-ie the effects of fading have to be compensated-with the aid of an estimate of the channel transfer function $H_{n}$. This estimate $\hat{H}_{n}$ can be obtained by the use of pilot subcarriers in the OFDM symbol, or by employing time-domain channel sounding training sequences embedded in the transmitted signal. Since the noise energy in each subcarrier is independent of the channel's frequency domain transfer function $H_{n}$, the local signal-to-noise ratio (SNR) in subcarrier $n$ can be expressed as

$$
\gamma_{n}=\left|H_{n}\right|^{2} \cdot \gamma
$$

where $\gamma$ is the overall SNR. If no signal degradation due to intersubcarrier interference (ISI) or interference from other sources appears, then the value of $\gamma_{n}$ determines the bit error probability for the transmission of data symbols over the subcarrier $n$.

The goal of adaptive modulation is to choose the appropriate modulation mode for transmission in each subcarrier, given the local SNR $\gamma_{n}$, in order to achieve a good tradeoff between throughput and overall BER. The acceptable overall BER varies depending on other systems parameters, such as the coding rate of the error correction coding, and the nature of the service supported by this particular link.

\section{B. Channel Model}

The impulse response $h(\tau, t)$ for the experiments was generated on the basis of the symbol-spaced impulse response shown in Fig. 3(a) by fading each of the impulses obeying a Rayleigh distribution of a normalized maximal Doppler frequency of $f_{d}^{\prime}=1.235 \cdot 10^{-5}$, where the normalization time duration was the length of the OFDM symbol, rather than the input bit duration. This is because the transceiver's performance 
is dependent on the Doppler-frequency related to the OFDM symbol duration. The above $f_{d}^{\prime}$ value corresponds to a wireless local area network (WLAN) channel experienced by a modem transmitting at a carrier frequency of $60 \mathrm{GHz}$ with a sample rate of $225 \mathrm{MHz}$ and a vehicular velocity of $50 \mathrm{~km} / \mathrm{h}$. The frequency domain complex channel transfer function $H_{n}$ corresponding to the unfaded impulse response is shown in Fig. 3(b).

\section{Channel Estimation}

The most conducive environment for an AOFDM scheme to operate in is a time division duplex (TDD) system in a slowly varying reciprocal channel, allowing open-loop adaptation. Both stations transmit an OFDM symbol in turn, and at each station, the most recent received symbol is used for the channel estimation, determining the modulation scheme adaptation for the next transmitted OFDM symbol. The channel estimation on the basis of the received symbol can be performed by pilot symbol assisted modulation (PSAM) [34], or upon invoking more sophisticated methods, such as decision-directed channel estimation. In this paper, we will initially assume perfect knowledge of the channel transfer function during the received timeslot.

\section{Choice of the Modulation Scheme}

The two communicating stations use the open-loop predicted channel transfer function acquired from the most recent received OFDM symbol, in order to allocate the appropriate modulation modes to the subcarriers. The modulation scheme was chosen from the set of Binary Phase Shift Keying (BPSK), Quadrature Phase Shift Keying (QPSK), 16-level Quadrature Amplitude Modulation (16-QAM), as well as "No Transmission," for which no signal was transmitted. These modulation schemes are denoted by $M_{m}$, where $m \in(0,1,2,4)$ is the number of data bits associated with a subcarrier.

In order to keep the system complexity low, the modulation scheme is not varied on a subcarrier-by-subcarrier basis, but instead the total OFDM bandwidth of 512 subcarriers is split into blocks of adjacent subcarriers, referred to as subbands, and the same modulation scheme is employed for all subcarriers of the same subband. This substantially simplifies the task of modem mode signaling and renders the employment of alternative blind detection mechanisms feasible, which will be discussed in Section II-E.

Three modulation scheme allocation algorithms were investigated in the subbands: a fixed threshold algorithm, an upperbound BER estimator and a fixed-throughput adaptation algorithm, which will be discussed below.

1) Fixed Threshold Adaptation Algorithm: The fixed threshold algorithm was derived from the adaptation algorithm proposed by Torrance for serial modems [35]. In the case of a serial modem, the channel quality is assumed to be constant for all symbols in the time slot, and hence the channel has to be slowly varying, in order to allow accurate channel quality prediction. Under these circumstances, all data symbols in the transmit time slot employ the same modulation scheme, chosen according to the predicted SNR. The SNR thresholds for a given long-term target BER were determined by Powell optimization [36]. Torrance assumed two uncoded target BERs:
TABLE I

OPTIMIZED SWITCHING LEVELS FOR ADAPTIVE MODULATION OVER RAYLEIGH FADING ChanNELS FOR THE "SPEECH" AND "DATA" SySteM, SHOWN IN INSTANTANEOUS CHANNEL SNR [dB] (FROM [36])

\begin{tabular}{l|c|c|c|c}
\hline & $l_{0}$ & $l_{1}$ & $l_{2}$ & $l_{4}$ \\
\hline speech system & $-\infty$ & 3.31 & 6.48 & 11.61 \\
\hline data system & $-\infty$ & 7.98 & 10.42 & 16.76 \\
\hline
\end{tabular}

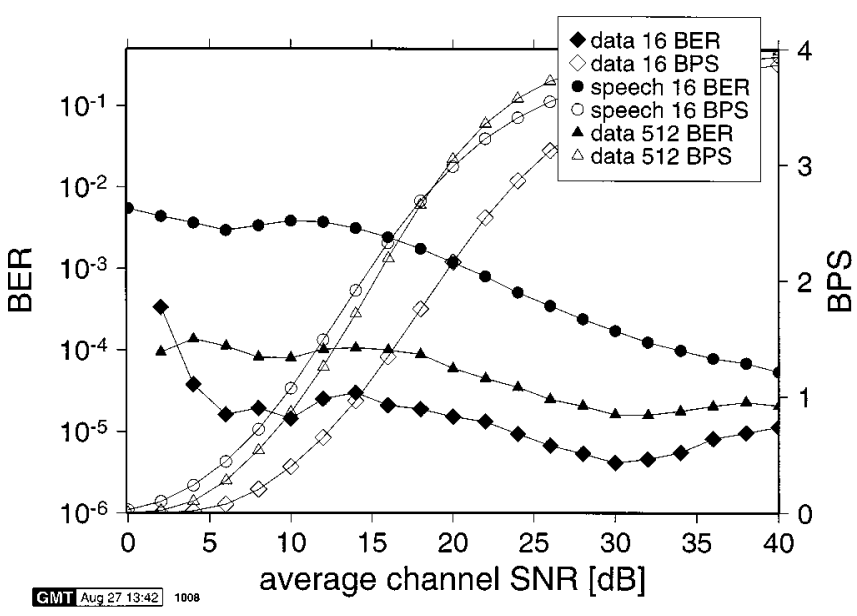

Fig. 4. BER and throughput performance of the 16 subband 512 subcarrier switching level adaptive OFDM modem employing BPSK, QPSK, 16-QAM and "no transmission" over the Rayleigh-fading time dispersive channel of Fig. 3.

$1 \%$ for a high data rate "speech" system, and $10^{-4}$ for a higher integrity, lower data rate "data" system. The resulting lower SNR thresholds $l_{n}$ for applying a given modulation scheme $M_{n}$ in a slowly Rayleigh fading narrow-band channel for both systems are given in Table I. The modulation scheme $M_{n}$ is selected, if the instantaneous channel SNR exceeds the switching level $l_{n}$.

Again, this adaptation algorithm assumed a constant instantaneous SNR over all of the block's symbols, but in the case of an OFDM system over a frequency selective channel the channel quality varies between the different subcarriers. For subband adaptive OFDM transmission this implies that if the subband width is wider, than the channel's coherence bandwidth, the above switching algorithm cannot be employed. For our investigations, we have therefore employed the lowest quality subcarrier in the subband for the adaptation based on the thresholds given in Table I. The performance of the 16 subband adaptive system over the WATM Rayleigh fading channel of Fig. 3 is shown in Fig. 4.

Adjacent or consecutive TDD timeslots have been used for the up- and downlink slots in these simulations, so that the delay between channel estimation and transmission was rendered as short as possible. Fig. 4 shows the long-term average BER and throughput of the studied modem for the "speech" and "data" switching levels of Table I, as well as for a subcarrier-by-subcarrier adaptive modem denoted by "data 512" employing the "data" switching levels. The results show the typical behavior of a variable-throughput adaptive system, which constitutes a tradeoff between best BER and throughput performance. For low SNR values, the system achieves a low BER by transmitting very few bits and only when the channel conditions allow. With 
increasing long-term SNR, the throughput rises, without significant change in the BER. For high SNR values the BER drops, as the throughput approaches its maximum of 4 bits per symbol (BPS), since the highest order constellation was 16 QAM.

It can be seen from the figure that the adaptive system performs better than its target BERs of $10^{-2}$ and $10^{-4}$ for the "speech" and "data" system, respectively, but that the measured BERs are lower than the targets. This can be explained by the adaptation regime, which was based on the conservative principle of invoking the lowest quality subcarrier in each subband for channel quality estimation, leading to a pessimistic channel quality estimate for the entire subband. For low values of SNR, the throughput in bits per data symbol is low and exceeds the fixed BPSK throughput of $1 \mathrm{bit} / \mathrm{symbol}$ only for SNR values of more than $9.5 \mathrm{~dB}$ and $14 \mathrm{~dB}$ for the "speech" and "data" systems, respectively.

The upper-bound performance of the system with subcarrier-by-subcarrier adaptation is also given in the figure, shown as 512 independent subbands, for the "data" optimized set of threshold values. It can be seen that in this case the target BER of $10^{-4}$ is very closely met over a wide range of SNR values from about $2-20 \mathrm{~dB}$, and that the throughput is considerably higher than in the case of the 16 subband modem. This is the result of more accurate subcarrier-by-subcarrier channel quality estimation and fine-grained adaptation, leading to better exploitation of the available channel capacity.

The throughput penalty of employing subband adaptation depends on the frequency-domain variation of the channel transfer function. If the subband bandwidth is lower than the channel's coherence bandwidth, then the assumption of constant channel quality per subband is reasonably met, and the system performance is equivalent to that of a subcarrier-by-subcarrier adaptive scheme.

2) Subband BER Estimator Adaptation Algorithm: We have seen above that the fixed switching level-based algorithm leads to a throughput performance penalty, if used in a subband adaptive OFDM modem, when the channel quality is not constant throughout each subband. This is due to the conservative adaptation based on the subcarrier experiencing the most hostile channel.

An alternative scheme taking into account the nonconstant SNR values $\gamma_{j}$ across the $N_{s}$ subcarriers in the $j$ th subband can be devised by calculating the expected overall bit error probability for all available modulation schemes $M_{n}$ in each subband, which is denoted by $\bar{p}_{e}(n)=1 / N_{s} \sum_{j} p_{e}\left(\gamma_{j}, M_{n}\right)$. For each subband, the scheme with the highest throughput, whose estimated BER is lower than a given threshold, is then chosen. While the adaptation granularity is still limited to the subband width, the channel quality estimation includes not only the worst subcarrier, which leads to an improved throughput.

Fig. 5 shows the BER and throughput performance for the 16 subband adaptive OFDM modem employing the BER estimator adaptation algorithm in the Rayleigh fading time dispersive channel of Fig. 3. The two sets of curves in the figure correspond to target BERs of $10^{-2}$ and $10^{-1}$, respectively. Comparing the modem's performance for a target BER of $10^{-2}$ with

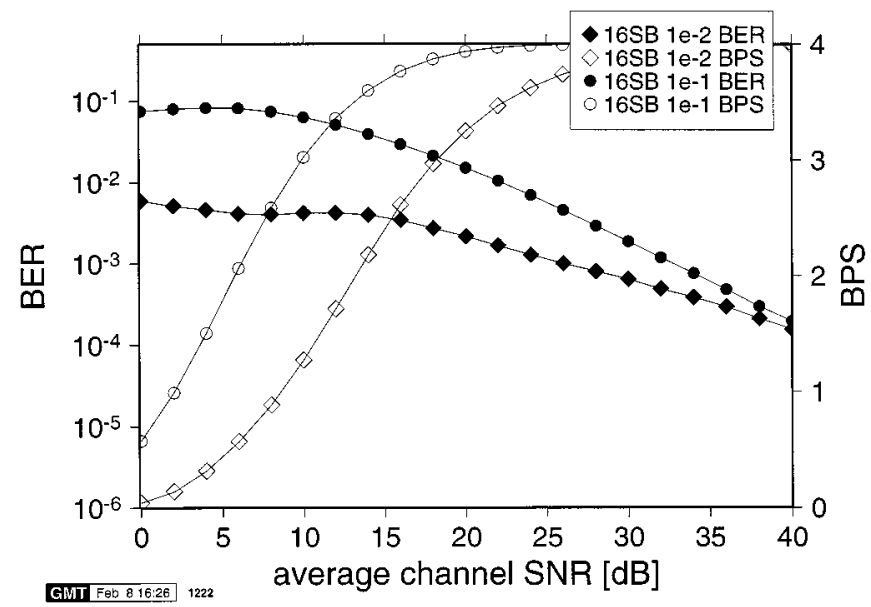

Fig. 5. BER and throughput performance of the 16 subband 512 subcarrier BER estimator adaptive OFDM modem employing BPSK, QPSK, 16-QAM and "no transmission" over the Rayleigh fading time dispersive channel of Fig. 3.

that of the "speech" modem in Fig. 4 it can be seen that the BER estimator algorithm results in significantly higher throughput, while meeting the BER requirements.

The BER estimator algorithm is readily adjustable to different target BERs, which is demonstrated in the figure for a target BER of $10^{-1}$. Such adjustability is beneficial, when combining adaptive modulation with channel coding, as it will be discussed in Section II-F.

3) Constant Throughput Adaptive OFDM: The time-varying data throughput of an adaptive OFDM modem operating with either of the two adaptation algorithms discussed above makes it difficult to employ such a scheme in a wide variety of applications. Torrance [35] studied the system implications of variable-throughput adaptive modems in the context of narrow-band channels, stressing the importance of data buffering at the transmitter, in order to accommodate the variable data rate. The required length of the buffer is related to the Doppler frequency of the channel, with a slowly varying channel resulting in slowly varying data throughput, and therefore the need for a high buffer capacity. Real-time audio or video transmission is very sensitive to delays, and therefore different adaptation algorithms are needed for such applications.

The constant throughput AOFDM scheme proposed here exploits the frequency selectivity of the channel, while offering a constant bit rate. Again, subband adaptivity is assumed, in order to simplify the signaling or the associated blind detection of the modulation schemes.

The modulation scheme allocation of the subbands is performed on the basis of a cost function, based on the expected number of bit errors in each subband. The expected number of bit errors, $e_{n, s}$, for each subband $n$ and each possible modulation scheme mode $s$, is calculated on the basis of the estimated channel transfer function $\hat{H}$, as well as a function of the number of bits transmitted per subband and modulation scheme, $b_{n, s}$.

Each subband is assigned a state variable $s_{n}$ holding the index of a modulation scheme. Each state variable is initialed to the 


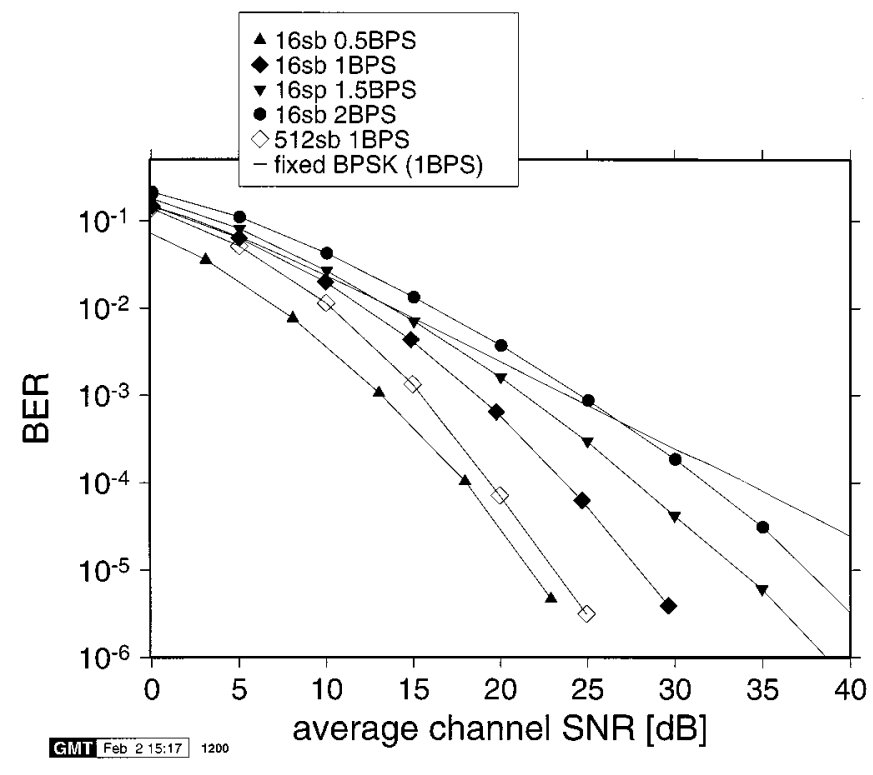

Fig. 6. BER performance versus SNR for the 512 subcarrier 16 subband constant throughput adaptive OFDM modem employing BPSK, QPSK, 16-QAM, and "no transmission" over the Rayleigh fading time dispersive channel of Fig. 3 for 0.5, 1, 1.5, and 2 BPS target throughput.

lowest order modulation scheme, which in our case is 0 for "no transmission." A set of cost values $c_{n, s}$ is calculated for each subband $n$ and state $s$ as follows:

$$
c_{n, s}=\frac{e_{n, s+1}-e_{n, s}}{b_{n, s+1}-b_{n, s}}
$$

for all but the highest level modulation index $s$. This cost value is related to the expected increase in the number of bit errors, divided by the increase of throughput, if the modulation scheme having the next higher index is used instead of index $s$ in subband $n$. In other words, (3) quantifies the expected incremental BER of the state transition $s \rightarrow s+1$ in subband $n$.

The modulation scheme adaptation is performed by repeatedly searching for the block $n$ having the lowest value of $c_{n, s_{n}}$, and incrementing its state $s_{n}$. This is repeated, until the total number of bits in the OFDM symbol reaches the target number of bits. Because of the granularity in bit numbers introduced by the subbands, the total number of bits may exceed the target. In this case, the data is padded with dummy bits for transmission.

Fig. 6 gives an overview of the BER performance of the fixed throughput (FT) 512 subcarrier OFDM modem in the time dispersive channel of Fig. 3 for a range of target bit numbers. The graph without markers represents the performance of a fixed BPSK OFDM modem over the same channel, which transmits 1 bit over each data subcarrier per OFDM symbol. The diamond-shaped markers give the performance of the equivalentthroughput adaptive scheme, both for the 16 subband arrangement in black, as well as for the subcarrier-by-subcarrier adaptive scheme in white. It can be seen that the 16 subband adaptive scheme yields a significant improvement in BER terms for SNR values above $10 \mathrm{~dB}$. The SNR gain for a BER of $10^{-4}$ is $8 \mathrm{~dB}$ compared to the nonadaptive case. Subcarrier-by-subcarrier adaptivity increases this gain by a further $4 \mathrm{~dB}$. The modem can easily be adapted to the system requirements by adjusting the target bit rate, as it is shown in Fig. 6. Halving the throughput to $0.5 \mathrm{BPS}$, the required SNR is reduced by $6 \mathrm{~dB}$ for a BER of $10^{-4}$, while increasing the throughput to 2 BPS deteriorates the noise resilience by $8 \mathrm{~dB}$ at the same BER.

\section{E. Signaling and Blind Detection}

The adaptive OFDM receiver has to be informed of the modulation schemes used for the different subbands. This information can either be conveyed using signaling subcarriers in the OFDM symbol itself, or the receiver can employ blind detection techniques in order to estimate the transmitted symbols' modulation schemes, as seen in Fig. 1.

1) Signaling: The simplest way of signaling the modulation scheme employed in a subband is to replace one data symbol by a $M$-PSK symbol, where $M$ is the number of possible modulation schemes. In this case, reception of each of the constellation points directly signals a particular modulation scheme in the current subband. In our case, for four modulation schemes, and assuming perfect phase recovery, the probability of a signaling error $p_{s}(\gamma)$, when employing one signaling symbol is the symbol error probability of QPSK. Then the correct subband mode signaling probability is

$$
\left(1-p_{s}(\gamma)\right)=\left(1-p_{b, \operatorname{QPSK}}(\gamma)\right)^{2}
$$

where $p_{b}, \mathrm{QPSK}$ is the bit error probability for QPSK

$$
p_{b, \mathrm{QPSK}}(\gamma)=Q(\sqrt{\gamma})=\frac{1}{2} \cdot \operatorname{erfc}\left(\sqrt{\frac{\gamma}{2}}\right)
$$

which leads to the expression for the modulation scheme signaling error probability of

$$
p_{s}(\gamma)=1-\left(1-\frac{1}{2} \cdot \operatorname{erfc}\left(\sqrt{\frac{\gamma}{2}}\right)\right)^{2} .
$$

The signaling error probability can be reduced by employing multiple signaling symbols and maximum ratio combining of the received signaling symbols $R_{s, n}$, in order to generate the decision variable $R_{s}^{\prime}$ prior to decision

$$
R_{s}^{\prime}=\sum_{n=1}^{N_{s}} R_{s, n} \cdot \hat{H}_{s, n}^{*}
$$

where

$N_{s} \quad$ number of signaling symbols per subband, the quantities;

$R_{s, n}$ received symbols in the signaling subcarriers;

$\hat{H}_{s, n}$ estimated values of the frequency domain channel transfer function at the signaling subcarriers.

Assuming perfect channel estimation and constant values of the channel transfer function across the group of signaling subcarriers, the signaling error probability for $N_{s}$ signaling symbols can be expressed as

$$
p_{s}^{\prime}\left(\gamma, N_{s}\right)=1-\left(1-\frac{1}{2} \cdot \operatorname{erfc}\left(\sqrt{\frac{N_{s} \gamma}{2}}\right)\right)^{2} .
$$

Fig. 7 shows the signaling error rate in an AWGN channel for $1,2,4$, and 8 signaling symbols per subband, respectively. It can be seen that doubling the number of signaling subcarriers 


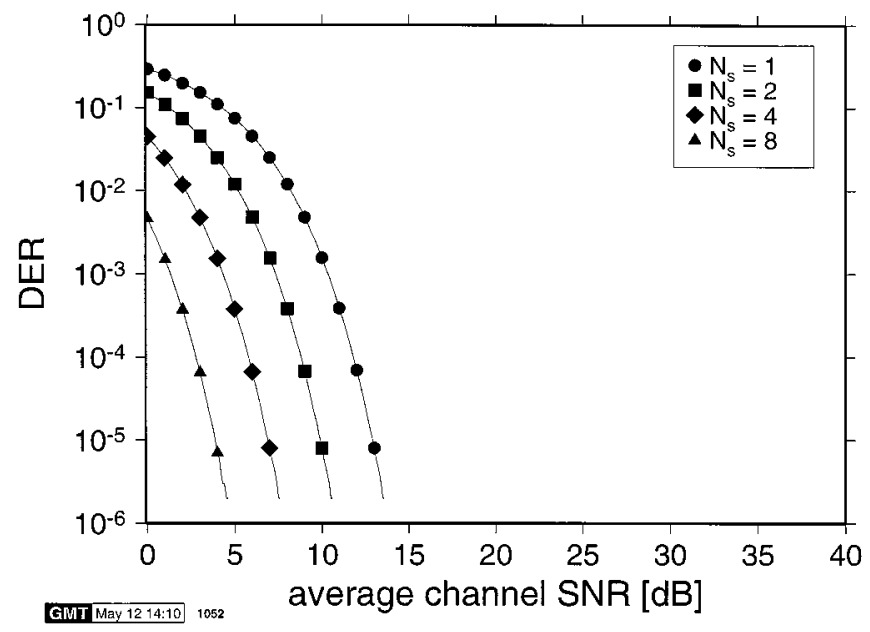

Fig. 7. Probability of erroneous modulation scheme detection, if signaling with maximum ratio combining is employed for QPSK symbols in an AWGN channel for 1,2, 4, and 8 signaling symbols per subband, evaluated from (8).

improves the performance by $3 \mathrm{~dB}$. Detection error rates (DERs) below $10^{-5}$ can be achieved at $10 \mathrm{~dB}$ SNR, if two signaling symbols are used. The signaling symbols for a given subband can be interleaved across the entire OFDM symbol bandwidth in order benefit from frequency diversity in fading wideband channels.

2) Blind Detection by SNR Estimation: The receiver has no a-priori knowledge of the modulation scheme employed in a particular received subband, and estimates this parameter by quantizing the de-faded-i.e., fading-compensated-received data symbols $R_{n} / \hat{H}_{n}$ in the subband to the closest symbol $\hat{R}_{n, m}$ for all possible modulation schemes $M_{m}$ for each subcarrier index $n$ in the current subband. The decision-directed error energy $e_{m}$ for each modulation scheme is calculated according to

$$
e_{m}=\sum_{n}\left(R_{n} / \hat{H}_{n}-\hat{R}_{n, m}\right)^{2}
$$

and the modulation scheme $M_{m}$, which minimizes $e_{m}$ is chosen for the demodulation of the subband.

The detection error probability of the blind modulation scheme detection algorithm described in this section for a 512 subcarrier OFDM modem in an AWGN channel is depicted in Fig. 8. It can be seen that the detection performance depends on the number of symbols per subband, with fewer subbands and therefore longer symbol sequences per subband leading to better detection performance. It is apparent, however, that the number of available modulation schemes has a more significant effect on the detection reliability than the block length. If all four legitimate modem modes are employed, then reliable detection of the modulation scheme is only guaranteed for SNR values of more than 15-18 dB, depending on the number of subbands per OFDM symbol. If only $M_{0}$ and $M_{1}$ are employed, however, the estimation accuracy is dramatically improved. In this case, SNR values above 5-7 dB are sufficient to ensure reliable detection.

Fig. 9 shows the BER performance of the fixed threshold "data"-type 16-subband adaptive system in the fading wide-

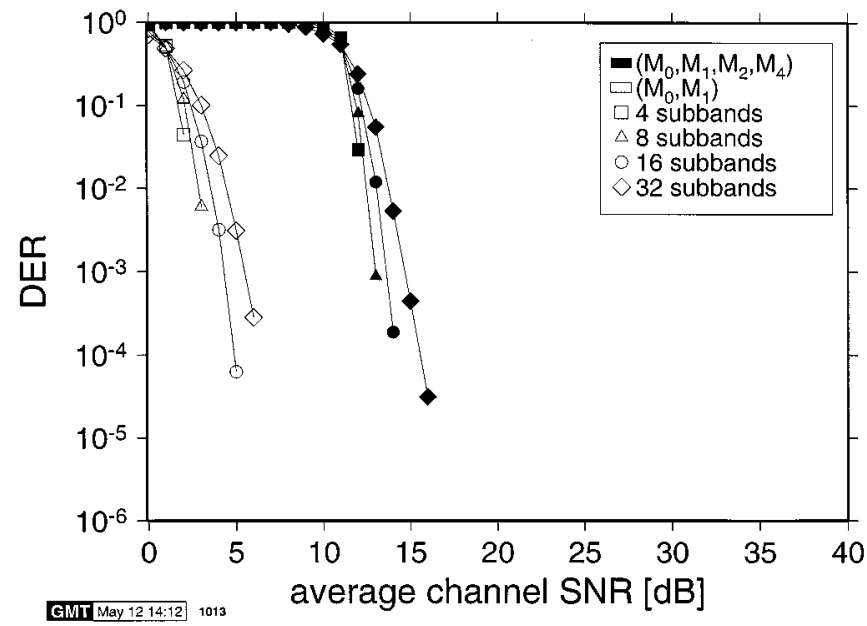

Fig. 8. Probability of erroneous blind modulation scheme detection for systems employing $\left(M_{0}, M_{1}\right)$ as well as for $\left(M_{0}, M_{1}, M_{2}, M_{4}\right)$ for different block lengths in an AWGN channel.

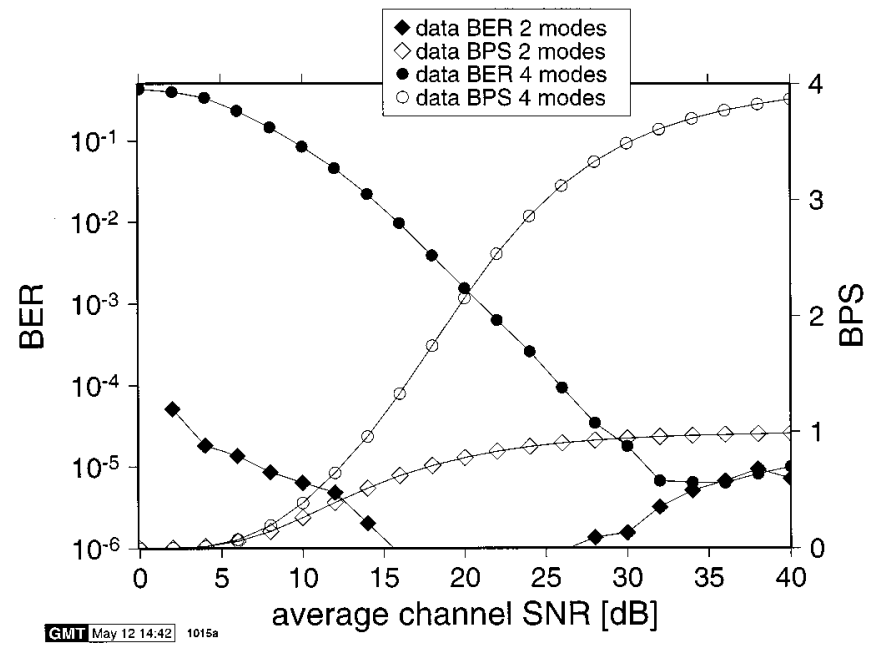

Fig. 9. BER and throughput performance of 16 subband 512 subcarrier adaptive OFDM modem employing No Transmission and BPSK (2 modes) or No Transmission, BPSK, QPSK and 16 QAM (4 modes), both for data-type switching levels and blind modulation scheme detection over the Rayleigh fading time-dispersive channel of Fig. 3.

band channel of Fig. 3 for both sets of modulation schemes, namely for $\left(M_{0}, M_{1}\right)$ and $\left(M_{0}, M_{1}, M_{2}, M_{4}\right)$ with blind modulation scheme detection. Erroneous decisions on the modulation scheme were assumed to yield a BER of 50\% in the received block. This is optimistic, since in a realistic scenario the receiver would have no knowledge of the number of bits actually transmitted, leading to loss of synchronization in the data stream. This problem is faced by all systems having a variable throughput and not employing an ideal reliable signaling channel. This impediment must be mitigated by data synchronization measures.

It can be seen from Fig. 9 that while blind modulation scheme detection yields poor performance for the quadruple-mode adaptive scheme, the twin-mode scheme exhibits very good BER results, consistently lower than $10^{-4}$.

3) Blind Detection by Multi-Mode Trellis Decoder: If error correction coding is invoked in the system, then the channel 


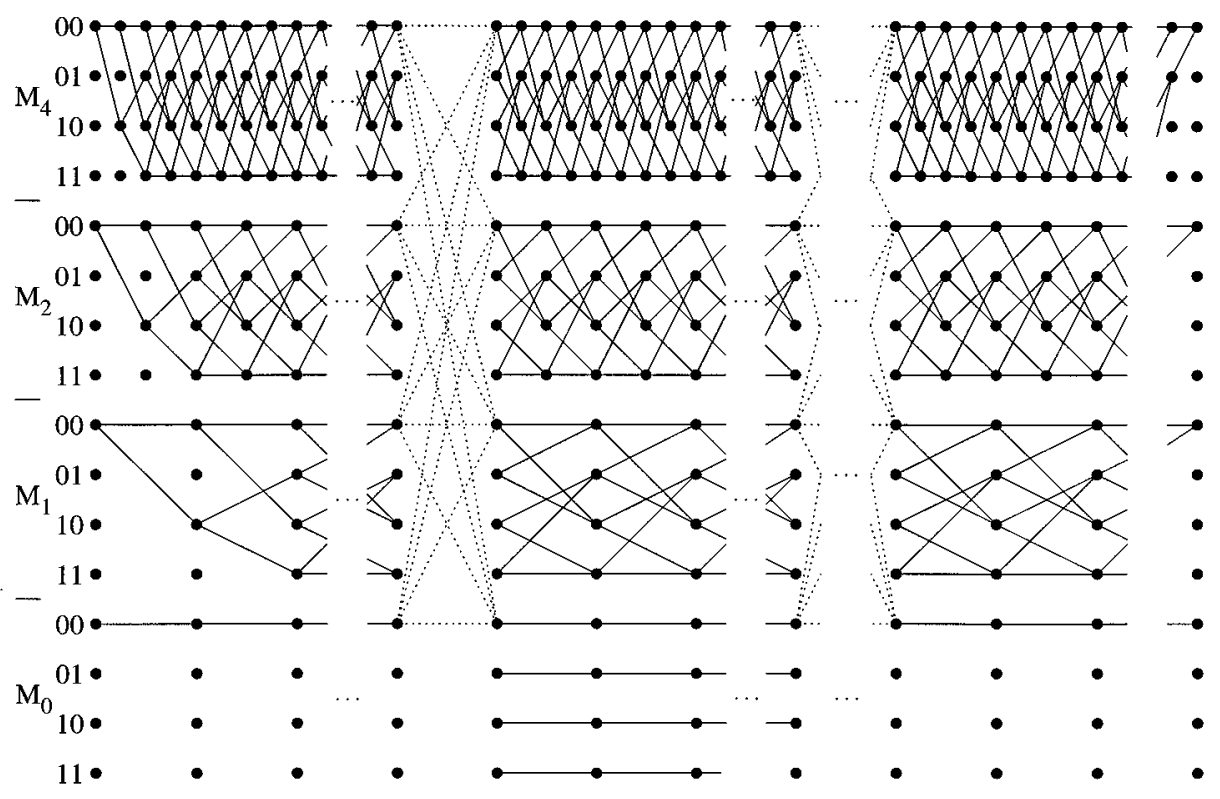

Fig. 10. Schematic plot of the parallel trellises for blind modulation scheme detection. In this example, a four-state 00-terminated convolution channel encoder was assumed. The dotted lines indicate the inter-subband transitions for the 00 state, and are omitted for the other three states.

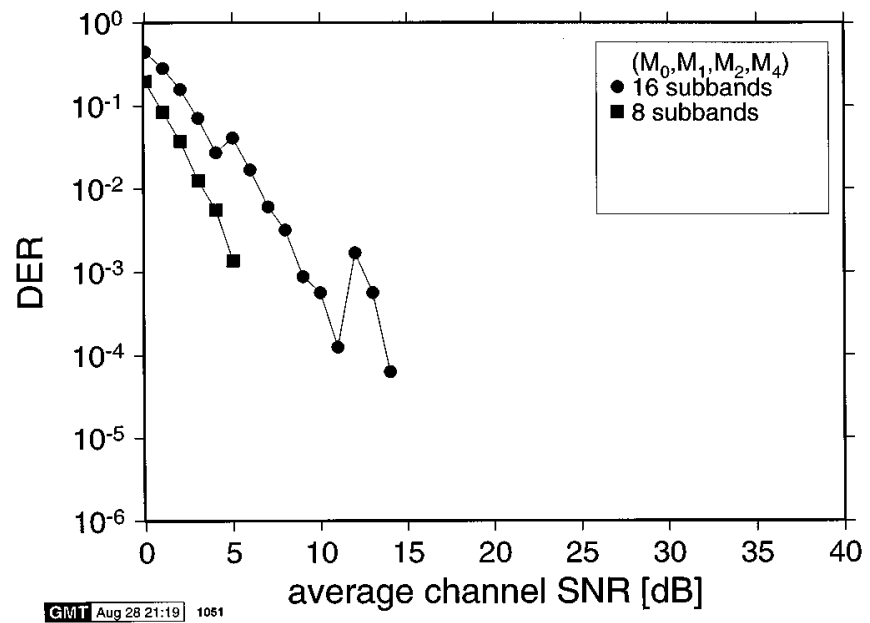

Fig. 11. Probability of erroneous blind modulation scheme detection using the "parallel trellis algorithm" with a $K=7$ convolutional code in an AWGN channel for a 512 subcarrier OFDM modem.

decoder can be employed to estimate the most likely modulation scheme per subband. Since the number of bits per OFDM symbol is varying in this adaptive scheme, and the channel encoder's block length therefore is not constant, for the sake of implementational convenience we have chosen a convolutional encoder at the transmitter. Once the modulation schemes to be used are decided upon at the transmitter, the convolutional encoder is employed to generate a zero-terminated code-word having the length of the OFDM symbol's capacity. This codeword is modulated on the subcarriers according to the different modulation schemes for the different subbands and transmitted over the channel.

At the receiver, each received data subcarrier is demodulated by all possible demodulators, and the resulting hard decision bits are fed into parallel trellises for Viterbi decoding. Fig. 10 shows a schematic sketch of the resulting parallel trellis if 16 QAM $\left(M_{4}\right)$, QPSK $\left(M_{2}\right)$, BPSK $\left(M_{1}\right)$, and "no transmission" $\left(M_{0}\right)$ are employed, for example for a code having four states. Each subband in the adaptive scheme corresponds to a set of four parallel trellises, whose inputs are generated independently by the four demodulators of the legitimate modulation schemes. The number of transitions in each of the trellises depends on the number of output bits received from the different demodulators, the 16 QAM $\left(M_{4}\right)$ trellis contains four times as many transitions as the BPSK and "no transmission" trellises. Since in the case of "no transmission" no coded bits are transmitted, the state of the encoder does not change. Therefore, legitimate transitions for this case are only horizontal ones.

At subband boundaries, transitions are allowed between the same state of all the parallel trellises associated with the different modulation modes. This is not a transition due to a received bit, and therefore preserves the metric of the originating state. Note that in the figure only the legitimate transitions for the state 00 are drawn; all other states originate the corresponding equivalent set of transitions. The initial state of the first subband is 00 for all modulation schemes, and, since the code is 00-terminated, the last subband's final states are 00 .

The receiver's channel decoder calculates the metrics for the transitions in the parallel trellises, and once all data symbol have been precessed, it traces back through the parallel trellis on the surviving path. This back-tracing commences at the most likely 00 state at the end of the last subband from the set of trellises associated with $M_{0} \cdots M_{4}$. If no termination was used at the decoder, then the back-tracing would start at the most likely of all the final states of the last block.

Fig. 11 shows the modulation scheme DER for the parallel trellis decoder in an AWGN channel for 16 and 8 subbands, if a convolutional code of constraint length 7 is used. Comparison with Fig. 8 shows considerable improvements relative to the BER-estimation-based blind detection scheme, both for 16 


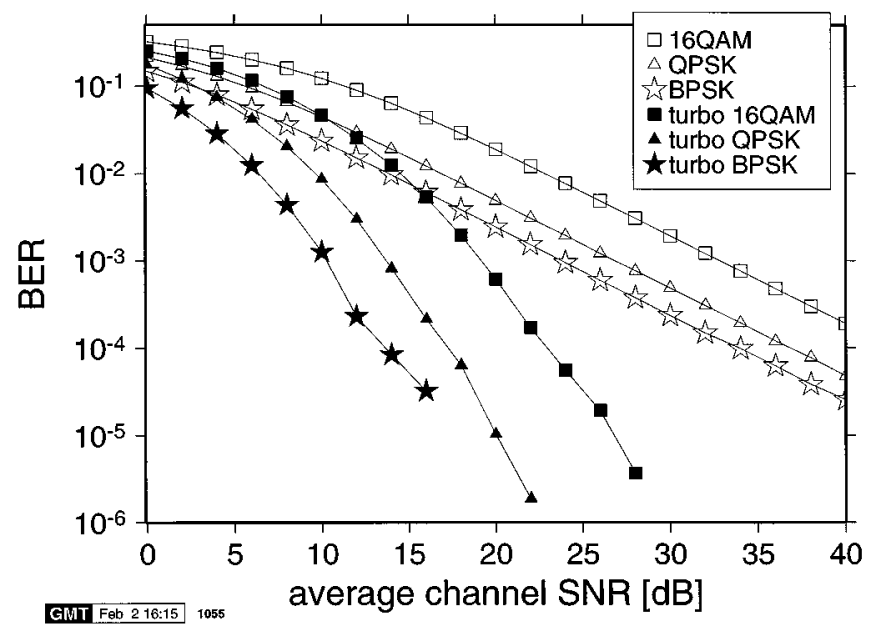

Fig. 12. BER performance of the 512-subcarrier OFDM modem in the fading time-dispersive channel of Fig. 3 for both uncoded and half-rate turbo-coded transmission, using 8-iteration turbo decoding, 1000-bit random interleaver and a constraint length of 3 .

as well as for 8 subbands. Higher subband lengths improve the estimation accuracy by a greater degree, than what has been observed for the BER estimation algorithm of Fig. 8. A DER of less than $10^{-5}$ was observed for an SNR value of 6 and 15 $\mathrm{dB}$ in the 8- and 16-subband scenarios, respectively. The use of stronger codes could further improve the estimation accuracy, at the cost of greater complexity.

\section{F. subband Adaptive OFDM and Channel Coding}

Adaptive modulation can reduce the BER to a level, where channel decoders can perform well. Fig. 12 shows both the uncoded and coded BER performance of a 512 subcarrier OFDM modem in the fading wideband channel of Fig. 3, assuming perfect channel estimation. The channel coding employed in this set of experiments is a turbo codec [37] with a data block length of 1000 bits, employing a random interleaver and 8 decoder iterations. Accordingly, interleaving and coding is applied to the entire OFDM symbol, rather than to the individual subbands. Combining the above turbo codec with adaptive modulation ensures that the modem loads the subcarriers with bits according to the associated subcarrier quality and hence a fixed-rate turbo codec is adequate for the protection of the bits. The constituent half-rate convolutional encoders were of constraint length 3 , with octally represented generator polynomials of $(7,5)$. It can be seen that the turbo decoder provides a considerable SNR gain for the different fixed modulation schemes, with a BER of $10^{-4}$ for SNR values of $13.8 \mathrm{~dB}, 17.3 \mathrm{~dB}$, and $23.2 \mathrm{~dB}$ for BPSK, QPSK, and 16 QAM transmission, respectively.

Fig. 13 depicts the BER and throughput performance of the same decoder employed in conjunction with the adaptive OFDM modem for different adaptation algorithms. Fig. 13(a) shows the performance for the "speech" system employing the switching levels listed in Table I. As expected, the half-rate channel coding results in a halved throughput compared to the uncoded case, but offers error-free transmission over the channel of Fig. 3 for SNR values of down to $0 \mathrm{~dB}$.

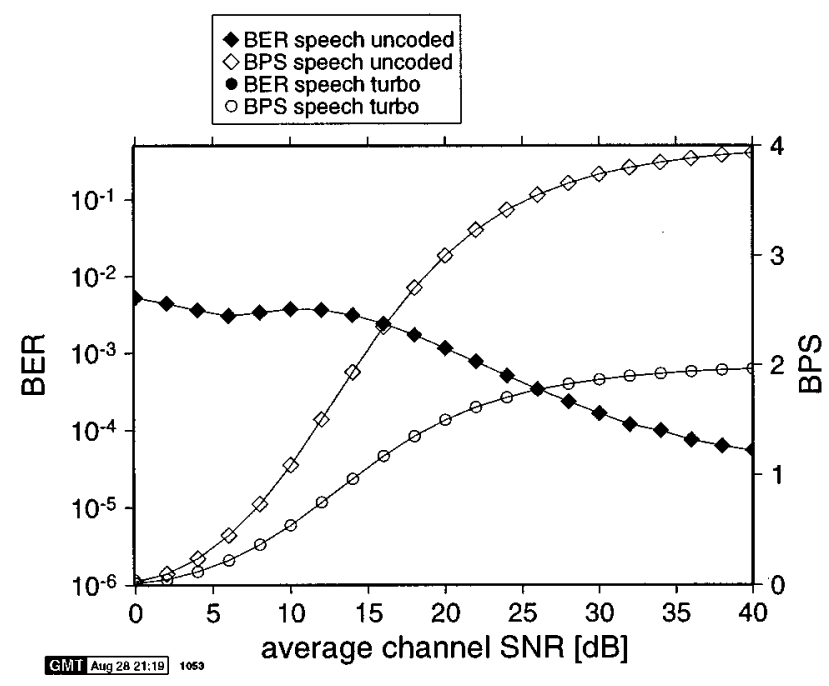

(a)

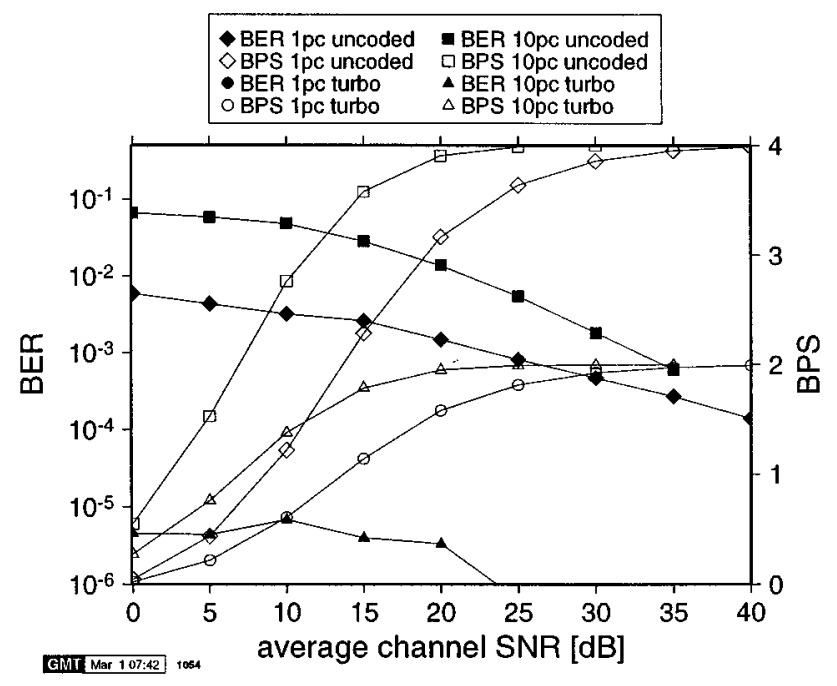

(b)

Fig. 13. BER and throughput performance of 16-subband 512-subcarrier adaptive turbo coded and uncoded OFDM modem employing $\left(M_{0}, M_{1}, M_{2}, M_{4}\right)$ for (a) speech type switching levels of Table I and (b) a maximal estimated subband BER of $1 \%$ and $10 \%$ over the channel of Fig. 3. The coded transmissions over the speech system and the $1 \%$ maximal BER system are error free for all examined SNR values, and therefore the corresponding BER curves are not visible in the figure.

Further tuning of the adaptation parameters can ensure a better average throughput, while retaining error-free data transmission. The switching level-based adaptation algorithm of Table I is difficult to control for arbitrary BERs, since the set of switching levels was determined by an optimization process. The subband BER predictor of Section II-D-2 is easier to adapt to a channel codec, and Fig. 13(b) shows the performance for the same decoder, with the adaptation algorithm employing the BER-prediction method having an upper BER-bound of $1 \%$. It can be seen that the less stringent uncoded BER constraints when compared to Fig. 13(a) lead to a significantly higher throughput for low SNR values. The decoded data bits are error-free, hence a further increase in throughput is possible.

The second set of curves in Fig. 13(b) show the system's performance, if an uncoded target BER of $10 \%$ is assumed. In this 


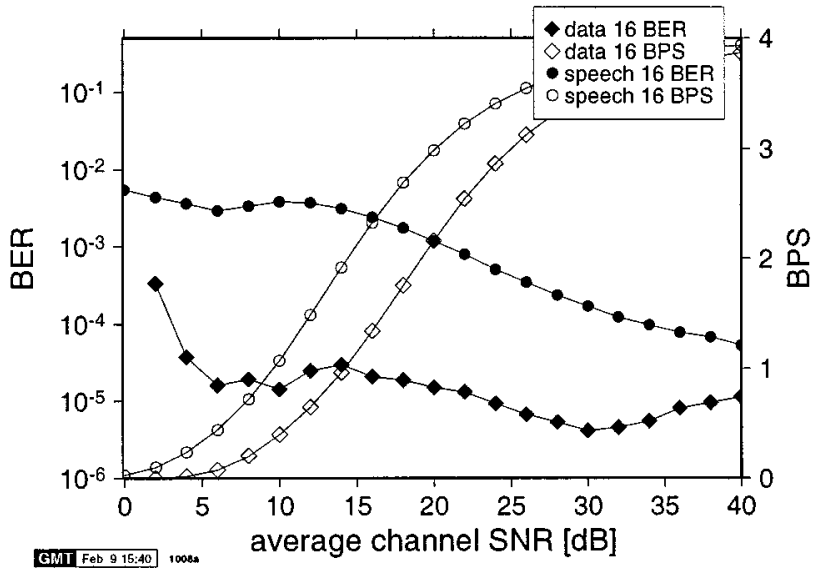

(a)

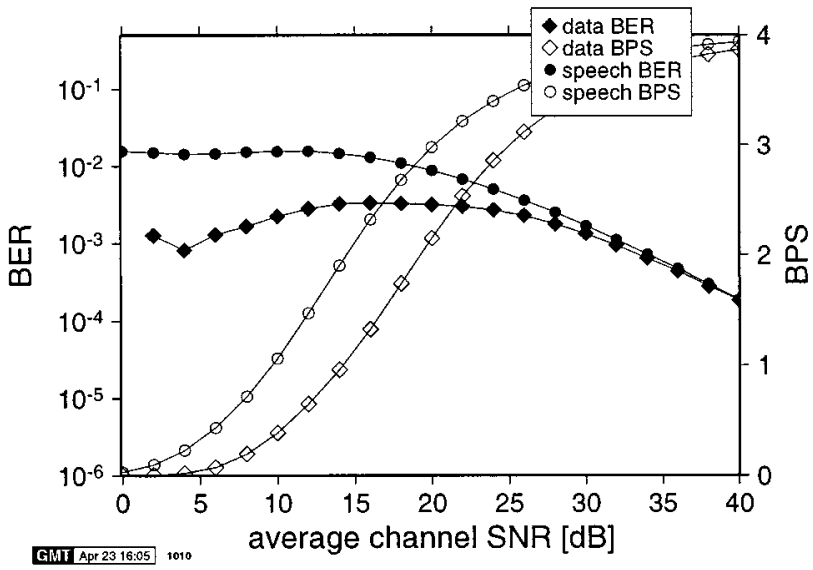

(c)

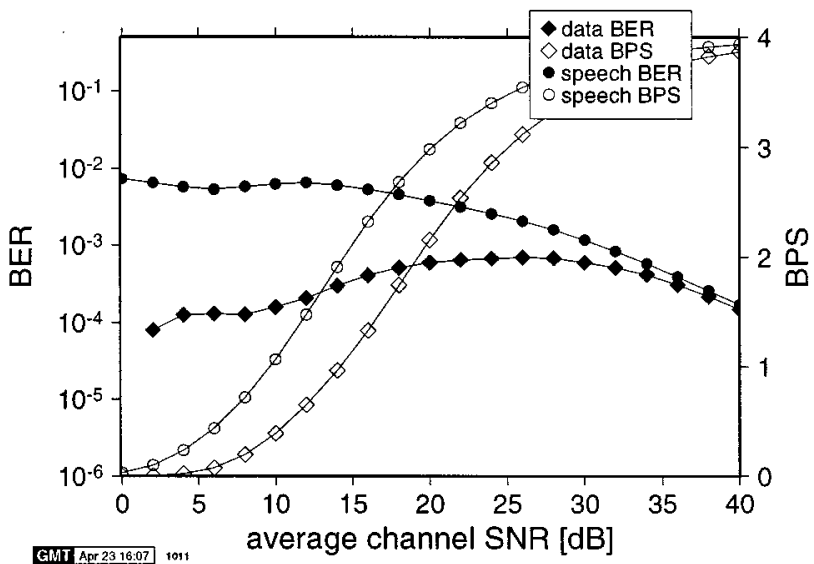

(b)

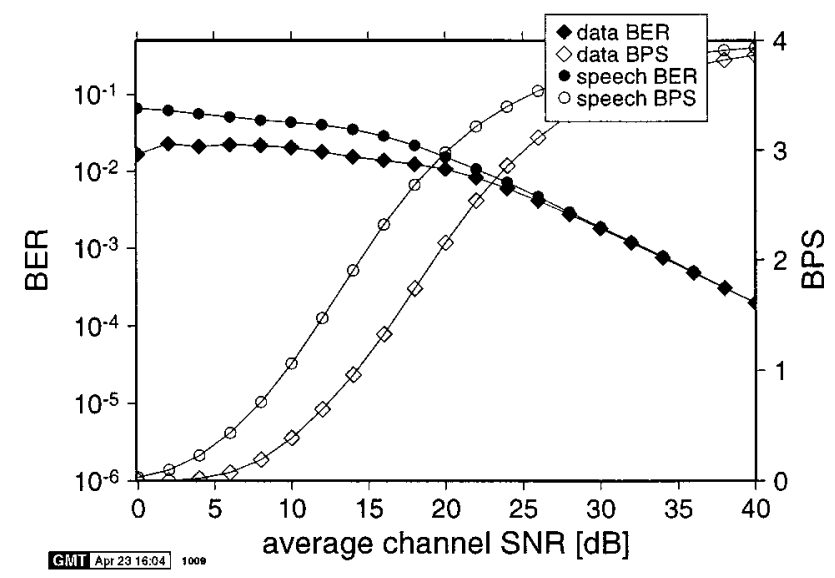

(d)

Fig. 14. BER and throughput performance of 16-subband 512-subcarrier adaptive OFDM modem employing $\left(M_{0}, M_{1}, M_{2}, M_{4}\right)$ for both data-type and speech-type switching levels and perfect modulation scheme detection as well as for different OFDM-symbol duration normalized Doppler frequencies $F_{d}^{\prime}$ over the channel of Fig. 3. The triangular markers in (a) show the performance of a subcarrier-by-subcarrier adaptive modem using the data-type switching levels of Table I for comparison.

case, the turbo decoder's output BER is below $10^{-5}$ for SNR values below $10 \mathrm{~dB}$ and shows a slow decrease for higher values of SNR. The throughput of the system, however, exceeds 0.5 data BPS for SNR values of more than $2 \mathrm{~dB}$.

\section{G. The Effect of Doppler Frequency}

Since the adaptive OFDM modem employs the most recently received OFDM symbol in order to predict the frequency domain transfer function of the reverse channel for the next transmission, the quality of this prediction suffers from changes of the channel transfer function between the uplink and downlink timeslots. We assume that the time delay between the upand downlink slots is the same as the delay between the downand uplink slots, and refer to this time as the frame duration $T_{f}$. We normalize the maximal Doppler frequency $f_{d}$ of the channel to the frame duration $T_{f}$, and define the frame-normalized Doppler frequency $F_{d}^{\prime}$ as $F_{d}^{\prime}=f_{d} \cdot T_{f}$. Fig. 14 depicts the fixed switching level (see Table I) modem's BER and throughput performance in bits-per-symbol (BPS) for values of $F_{d}^{\prime}$ between $7.41 \cdot 10^{-3}$ and $2.3712 \cdot 10^{-1}$. These values stem from the studied WLAN system with a time slot duration of $2.67 \mu$ s and up-/downlink delays of $1,8,16$, and 32 timeslots at a channel Doppler frequency of $2.78 \mathrm{kHz}$. As mentioned in Section II-B, this corresponds to a system employing a carrier frequency of $60 \mathrm{GHz}$, a sampling rate of $225 \mathrm{M}$ Samples/s and a vehicular velocity of $50 \mathrm{~km} / \mathrm{h}$ or $13 . \overline{8} \mathrm{~m} / \mathrm{s}$.

Fig. 14(a) shows the BER and throughput of the studied modems in a framework with consecutive up- and downlink timeslots. This corresponds to $F_{d}^{\prime}=7.41 \cdot 10^{-3}$, while the target BERs for the speech and data system are met for all SNR values above $4 \mathrm{~dB}$, and the BER performance is generally better than the target error rates. This was explained above with the conservative choice of modulation schemes based on the most corrupted subcarrier in each subband, resulting in lower throughput and lower BERs for the switching level-based subband adaptive modem.

Comparing Fig. 14(a) with the other performance curves, it can be seen that the BER performance for both the speech and the data system suffer from increasing decorrelation of the predicted and actual channel transfer function for increasing values of $F_{d}^{\prime}$. In Fig. 14(b) an eight time-slot delay was assumed between the up- and downlink timeslots, which corresponds to $F_{d}^{\prime}=5.928 \cdot 10^{-2}$, and the BER performance of the modem significantly deteriorated. The "speech" system still maintains its target BER, but the "data" system delivers a BER of up to 


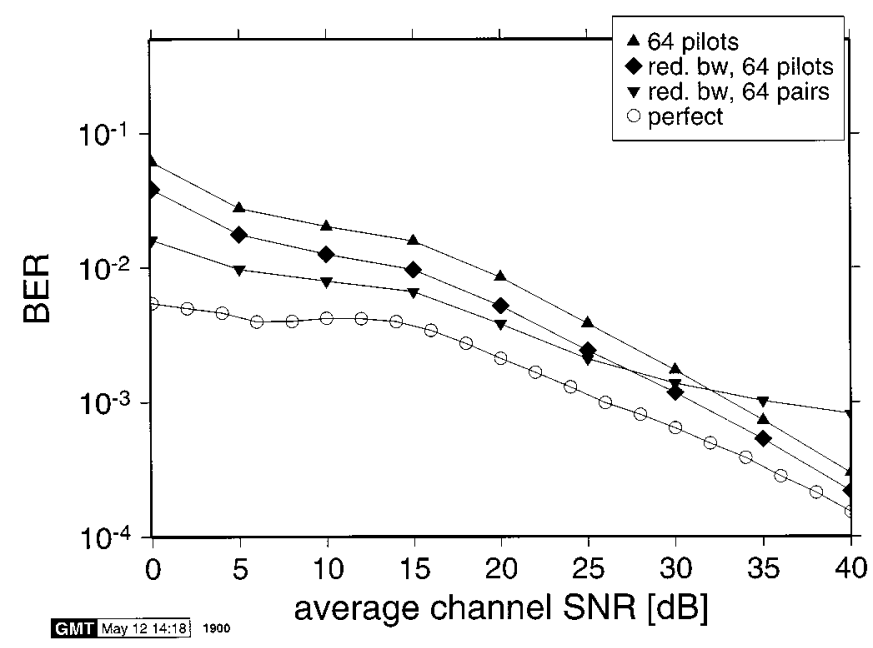

Fig. 15. BER versus channel SNR performance for the $1 \%$ target BER adaptive 16-subband 512-subcarrier OFDM modem employing pilot symbol assisted channel estimation over the channel of Fig. 3.

$10^{-3}$ for SNR values between 25 and $30 \mathrm{~dB}$. It is interesting to observe that the delayed channel prediction mainly affects the higher order modulation schemes, which are employed more frequently at high SNR values. This explains the shape of the BER curve for the "data" system, which is rising from below $10^{-4}$ at $2 \mathrm{~dB}$ SNR up to $10^{-3}$ at $26 \mathrm{~dB}$ SNR. The average throughput of the modem is determined by the statistics of the estimated channel transfer function at the receiver, and this is therefore not affected by the delay between the channel estimation and the packet transmission.

\section{H. Channel Estimation}

All the adaptive modems above rely on the estimate of the frequency-domain channel transfer function, both for equalization of the received symbols at the receiver, as well as for the modem mode adaptation of the next transmitted packet. Fig. 15 shows the BER versus SNR curves for the $1 \%$ target-BER modem, as it was presented above, if pilot symbol assisted channel estimation [35] is employed instead of the previously used delayed-but otherwise perfect-channel estimation.

Comparing the curves for perfect channel estimation and for the 64-pilot-based lowpass interpolation algorithm, it can be seen that the modem falls short of the target BER of $1 \%$ for channel SNR values of up to $20 \mathrm{~dB}$. More noise-resilient channel estimation algorithms can improve the modem's performance. If the pass-band width of the interpolation lowpass filter is halved, which is indicated in Fig. 15 as the reduced bandwidth (red. bw.) scenario, then the BER gap between the perfect and the pilot symbol assisted channel estimation narrows, and a BER of $1 \%$ is achieved at an SNR of 15 $\mathrm{dB}$. Additionally, employing pairs of pilots with the above bandwidth-limited interpolation scheme further improves the modem's performance, which results in BER figures of below $1 \%$ for SNR values above $5 \mathrm{~dB}$. The averaging of the pilot pairs improves the noise resilience of the channel estimation, but introduces estimation errors for high SNR values. This can be observed in the residual BER in the figure.

\section{AdAPtive Modulation with SPECTRAl Pre-Distortion}

We have seen above, how the estimate of the channel transfer function at the transmitter can be employed in order to dramatically improve the performance of an OFDM system by adapting the subcarrier modulation schemes to the channel conditions. For subchannels exhibiting a low SNR, robust modulation schemes were used, while for subcarriers having a high SNR, high throughput multi-level modulation schemes can be employed. The performance of adaptive OFDM modems can be increased by using the knowledge of the channel's transfer function for spectral predistortion at the transmitter.

As discussed in the context of (1), the received data symbol $R_{n}$ of subcarrier $n$ over a stationary time-dispersive channel is characterized by the product of the transmitted symbol $S_{n}$ and the channel transfer function $H_{n}$ in subcarrier $n$, plus an additional noise sample $n_{n}$.

Equalization at the receiver, which is necessary for nondifferential detection of the data symbols, corrects the phase and amplitude of the received data symbols using the estimate of the channel transfer function $\hat{H}_{n}$ as follows:

$$
R_{n}^{\prime}=R_{n} / \hat{H}_{n}=S_{n} \cdot H_{n} / \hat{H}_{n}+n_{n} / \hat{H}_{n}
$$

If the estimate $\hat{H}_{n}$ is accurate, this operation reconstructs the constellation points before decision. However, the noise sample $n_{n}$ is amplified by the same amount as the signal, therefore preserving the SNR of the received sample.

Preequalization for the OFDM modem operates by scaling the data symbol of subcarrier $n, S_{n}$, by a predistortion function $E_{n}$, computed from the inverse of the estimated channel transfer function prior to transmission. At the receiver no equalization is performed, hence the received symbols can be expressed as:

$$
R_{n}=S_{n} \cdot E_{n} \cdot H_{n}+n_{n}
$$

Since no equalization is performed, there is no noise amplification at the receiver. Similarly to the adaptive modulation techniques illustrated above, pre-equalization is only applicable to a duplex link, since the transmitted signal is adapted to the specific channel conditions perceived by the receiver. Like for other adaptive schemes, the transmitter needs an estimate of the current frequency-domain channel transfer function, which can be obtained from the received signal in the reverse link, as seen in Fig. 1.

Combining the target-BER adaptive modulation scheme with spectral pre-distortion allows the transmitter to react to the channel's time- and frequency-variant nature, in order to fine-tune the behavior of the adaptive modem in fading channels. It also allows the transmitter to invest the energy that is not used in "no transmission" subbands into the other subbands without affecting the equalization at the receiver.

The aim of the joint modulation adaptation and pre-distortion algorithm is to transmit a subband's data symbols at a power level, which ensures a given target SNR at the receiver. The target SNR requirements depend on the acceptable BER of the link and the modulation scheme in use. Clearly, the receiver has to anticipate the different relative power levels for the different 
TABLE II

REQUIRED TARGET SNR LEVELS FOR $0.01 \%, 1 \%$ AND $10 \%$ TARGET BER FOR THE DIFFERENT MODULATION SCHEMES OVER AN AWGN CHANNEL

\begin{tabular}{l|c|c|c|c}
\hline & $l_{0}$ & $l_{1}$ & $l_{2}$ & $l_{4}$ \\
\hline speech system & $-\infty$ & 3.31 & 6.48 & 11.61 \\
\hline data system & $-\infty$ & 7.98 & 10.42 & 16.76 \\
\hline
\end{tabular}

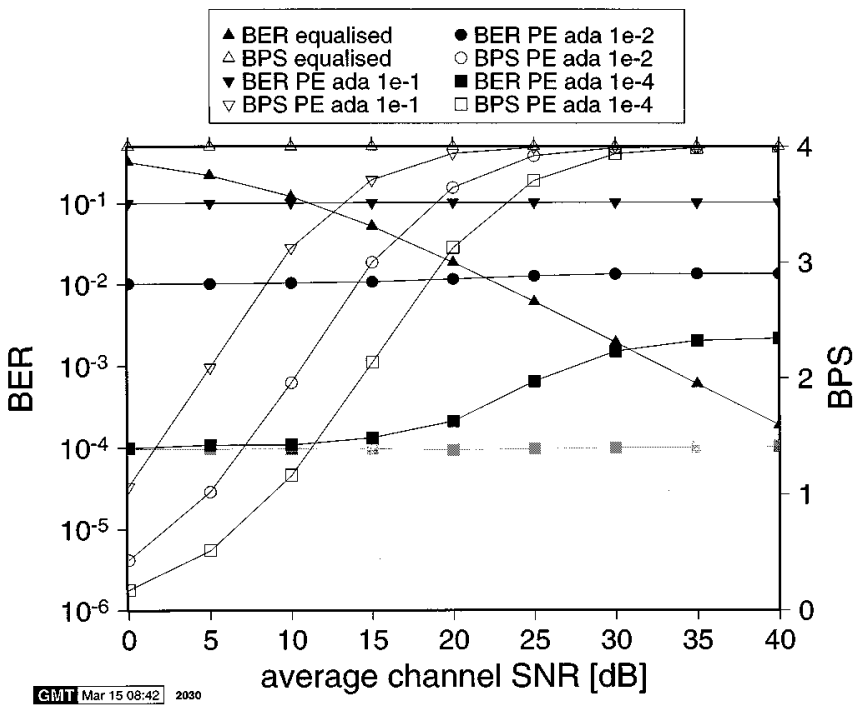

Fig. 16. BER performance and throughput of the 16 subband adaptive OFDM modem with spectral preequalization over the Rayleigh fading time dispersive short WATM channel of Fig. 3, and that of the perfectly equalized 16-QAM modem. The gray BER curve gives the performance of the adaptive modem for a target BER of $10^{-4}$ with no delay between channel estimation and transmission, while the other results assume one timeslot delay between up- and downlink.

modulation schemes, so that demodulation of the multi-level modulation schemes employed becomes possible.

The joint adaptation algorithm requires the estimates of the noise floor level at the receiver as well as the channel transfer function, which includes the path-loss. On the basis of these values, the necessary amplitude of $E_{n}$ required to transmit a data symbol at the subcarrier $n$ for a given received SNR of $\gamma_{n}$ can be calculated as follows:

$$
\left|E_{n}\right|=\frac{\sqrt{N_{0} \cdot \gamma_{n}}}{\left|\hat{H}_{n}\right|}
$$

where $N_{0}$ is the noise floor at the receiver. The phase of $E_{n}$ is used for the pre-equalization, and hence:

$$
\angle E_{n}=-\angle \hat{H}_{n} .
$$

The target SNR of subcarrier $n, \gamma_{n}$, is dependent on the modulation scheme that is used for the subcarrier, and determines the system's target BER. We have identified three sets of target SNR values for the modulation schemes, with uncoded target BER values of $1 \%$ and $10 \%$ for use in conjunction with channel coders, as well as $10^{-4}$ for transmission without channel coding. Table II gives an overview of these levels, which have been read from the BER performance curves for the different modulation schemes in a Gaussian channel.

Fig. 16 shows the performance of the joint pre-distortion and adaptive modulation algorithm in the fading time-dispersive

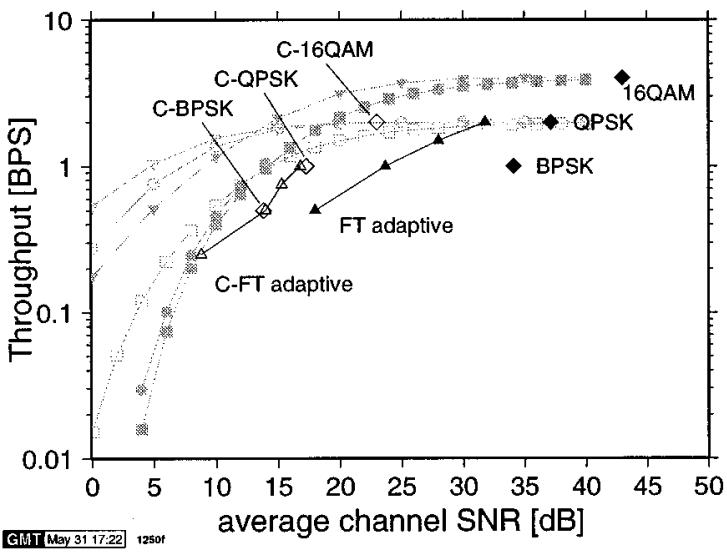

(a)

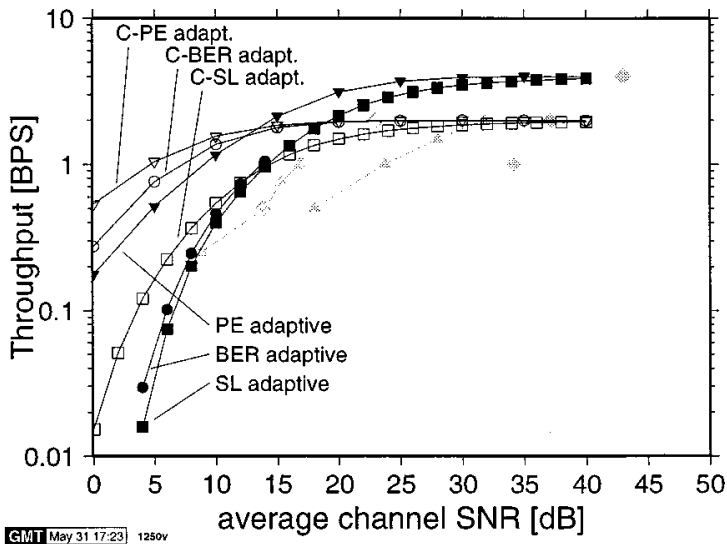

(b)

Fig. 17. Throughput versus average channel SNR for nonadaptive and adaptive modulation as well as for preequalized (PE) adaptive techniques, for a data BER of $10^{-4}$. Note that for the coded schemes the achieved BER values are lower than $10^{-4}$ : (a) FT systems: coded (C-) and uncoded BPSK, QPSK, 16 QAM, and FT adaptive modulation and (b) variable throughput systems: coded $(\mathrm{C}-$ ) and uncoded switching level adaptive (SL), target-BER adaptive (BER) and preequalized adaptive (PE) systems. Note that the separately plotted variable-throughput graph also shows the lightly shaded benchmarker curves of the fixed-rate schemes and vice versa.

short WATM channel of Fig. 3 for the set of different target BER values of Table II, as well as the comparison curves of the perfectly equalized 16-QAM modem using channel inversion under the same channel conditions. It can be seen that the BER achieved by the system is close to the BER targets. Specifically, for a target BER of $10 \%$, no perceptible deviation from the target has been recorded, while for the lower BER targets the deviations increase for higher channel SNRs. For a target BER of $1 \%$, the highest measured deviation is at the SNR of $40 \mathrm{~dB}$, where the recorded BER is $1.36 \%$. For the target BER of $10^{-4}$, the BER deviation is small at $0 \mathrm{~dB}$ SNR, but at an SNR of $40 \mathrm{~dB}$ the experimental BER is $2.2 \cdot 10^{-3}$. This increase of the BER with increasing SNR is due to the rapid channel variations in the deeply faded subcarriers, which are increasingly used at higher SNR values. The gray curve in the figure denotes the system's performance, if no delay is present between the channel estimation and the transmission. In this case, the simulated BER shows only very little deviation from the target BER value. This is consistent with the behavior of the full channel inversion pre-equalizing modem. 


\section{COMPARISON OF THE ADAPTIVE TECHNIQUES AND CONCLUSIONS}

Fig. 17 compares the different adaptive modulation schemes discussed in this contribution. The comparison graph is split into two sets of curves, depicting the achievable data throughput for a data BER of $10^{-4}$ highlighted for the FT systems in Fig. 17(a), and for the varying-throughput systems in Fig. 17(b).

The FT systems, highlighted in Fig. 17(a) comprise the 1, 2, and 4 bit/symbol nonadaptive BPSK, QPSK, and 16 QAM modems, as well as the fixed-but programmable $0.5,1,1.5$ and 2 bit/symbol throughput adaptive scheme, both for coded and uncoded applications. The corresponding throughput of the coded schemes was reduced to $0.25,0.5,0.75$, and $1 \mathrm{bit} / \mathrm{symbol}$. The nonadaptive modems' performance is marked on the graph as diamonds, and it can be seen that the uncoded fixed schemes require the highest channel SNR of all examined transmission methods, in order to achieve a data BER of $10^{-4}$. Again, channel coding employing the advocated turbo coding schemes dramatically improves the SNR requirements, at the expense of halving the data throughput. The uncoded FT adaptive schemes indicated by the hollow diamonds, marked by filled triangles, yields consistently worse data throughput than the coded fixed modulation schemes, with its throughput being half of the uncoded fixed scheme's at the same SNR values. The coded FT-adaptive system, however, delivers very similar throughput to the coded BPSK and coded QPSK transmission, and can deliver a BER of $10^{-4}$ for lower SNR values.

The variable throughput schemes, highlighted in Fig. 17(b), outperform the comparable FT algorithms. For high SNR values, all uncoded schemes' performance curves converge to a throughput of $4 \mathrm{bits} / \mathrm{symbol}$, which is equivalent to 16 QAM transmission. The coded schemes reach a maximal throughput of 2 bits/symbol. Of the uncoded schemes, the "data" switching-level (SL) and target-BER adaptive modems deliver a very similar BPS performance, with the target-BER scheme exhibiting slightly better throughput than the SL adaptive modem. The adaptive modem employing pre-equalization (PE) significantly outperforms the other uncoded adaptive schemes, and offers a throughput of $0.18 \mathrm{BPS}$ at an SNR of $0 \mathrm{~dB}$.

The coded transmission schemes suffer from limited throughput at high SNR values, as the half-rate channel coding limits the data throughput to 2 BPS. For low SNR values, however, the coded schemes offer better performance than the uncoded schemes, with the exception of the "speech" SL-adaptive coded scheme, which is outperformed by the uncoded PE-adaptive modem. The poor performance of the coded SL-scheme can be explained by the lower uncoded target BER of the "speech" scenario, which was $1 \%$, in contrast to the $10 \%$ uncoded target BER for the coded BER- and PE-adaptive schemes. The coded PE-adaptive modem outperforms the target-BER adaptive scheme, thanks to its more accurate control of the uncoded BER, leading to a higher throughput for low SNR values.

It is interesting to observe that for the given set of four modulation schemes the uncoded PE-adaptive scheme is close in performance to the coded adaptive schemes, and that for SNR values of more than $14 \mathrm{~dB}$ it outperforms all other studied schemes. It is clear, however, that the coded schemes would benefit from higher order modulation modes, which would allow these modems to increase the data throughput further. Our future work is targeted at invoking turbo-decoded, maximum minimum distance so-called redundant residual number system channel codes in conjunction with hybrid Automatic repeat request (ARQ) schemes of type II, in an effort to increase the system's performance.

\section{REFERENCES}

[1] R. Steele and W. Webb, "Variable rate QAM for data transmission over Rayleigh fading channels," in Wireless. Calgary, Alberta, Canada: IEEE, 1991, pp. 1-14.

[2] L. Hanzo, W. T. Webb, and T. Keller, Single and Multi-Carrier Quadrature Amplitude Modulation. New York: Wiley-IEEE Press, 2000.

[3] H. Matsuoka, S. Sampei, N. Morinaga, and Y. Kamio, "Adaptive modulation system with variable coding rate concatenated code for high quality multi-media communications systems," in 46th Vehicular Technology Conf.. Piscataway, NJ: IEEE, 1996, pp. 478-491.

[4] S.-G. Chua and A. Goldsmith, "Variable-rate variable-power MQAM for fading channels," in 46th Vehicular Technology Conf.. Piscataway, NJ: IEEE, 1996, pp. 815-819.

[5] J. M. Torrance and L. Hanzo, "Latency and networking aspects of adaptive modems over slow indoors rayleigh fading channels," IEEE Trans. Veh. Technol., vol. 48, pp. 1237-1251, July 1999.

[6] J. M. Torrance, L. Hanzo, and T. Keller, "Interference aspects of adaptive modems over slow Rayleigh fading channels," IEEE Trans. Veh. Technol., vol. 48, pp. 1527-1545, Sept. 1999.

[7] T. H. Liew, C.H. Wong, and L. Hanzo, "Block turbo coded burst-by-burst adaptive modems," in Proc. Microcoll'99, Budapest, Hungary, March 21-24, 1999, pp. 59-62.

[8] C. H. Wong, T. H. Liew, and L. Hanzo, "Blind modem mode detection aided block turbo coded burst-by-burst wideband adaptive modulation," in Proc. of ACTS'99, Sorrento, Italy, June 8-11, 1999, Paper no. 10 254, to be published.

[9] M. S. Yee and L. Hanzo, "Upper-bound performance of radial basis function decision feedback equalized burst-by-burst adaptive modulation," in Proc. ECMCS'99, Krakow, Poland, June 24-26, 1999.

[10] V. K. N. Lau and M. D. Macleod, "Variable rate adaptive trellis coded QAM for high bandwidth efficiency applications in Rayleigh fading channels," in Proc. IEEE Vehicular Technology Conf., 1998, pp. $348-352$.

[11] S. G. Chua and A. Goldsmith, "Adaptive coded modulation for fading channels," IEEE Trans. Commun., vol. 46, pp. 595-602, May 1998.

[12] T. Keller and L. Hanzo, "Adaptive orthogonal frequency division multiplexing schemes," in Proc. ACTS Summit, Rhodos, Greece, June 1998, pp. 794-799.

[13] E. L. Kuan, C. H. Wong, and L. Hanzo, "Burst-by-burst adaptive joint detection CDMA," in Proc. VTC'99, Houston, TX, May 1998, pp. $1628-1632$.

[14] T. Keller, J. Woodard, and L. Hanzo, "Turbo-coded parallel modem techniques for personal communications," in Proc. 47th Vehicular Technology Conf.. Phoenix, AZ: IEEE, May 5-7, 1997, pp. 2158-2162.

[15] P. Cherriman, T. Keller, and L. Hanzo, "Constant-rate turbo-coded and block-coded orthogonal frequency division multiplex videophony over UMTS," in Proc. Globecom'98, Sydney, Australia, Nov. 1998, pp. 2848-2852.

[16] J. P. Woodard, T. Keller, and L. Hanzo, "Turbo-coded orthogonal frequency division multiplex transmission of 8 kbps encoded speech," in Proc. ACTS'97, Aalborg, Denmark, Oct. 1997, pp. 894-899.

[17] P. R. K. Fazel, S. Kaiser, and M. Ruf, "A concept of digital terrestrial television broadcasting," Wireless Personal Communications, vol. 2, pp. 9-27, 1995.

[18] I. J. Hikmet Sari and G. Karam, "Transmission techniques for digital terrestrial tv broadcasting," IEEE Commun. Mag., Feb. 1995.

[19] J. Borowski, S. Zeisberg, J. Hübner, K. Koora, E. Bogenfeld, and B. Kull, "Performance of OFDM and comparable single carrier system in MEDIAN demonstrator 60GHz channel," in Proc. ACTS Summit, Aalborg, Denmark, Oct. 1997, pp. 653-658.

[20] B. Engstroem and C. Oesterberg, "A system for test of multiaccess methods based on OFDM," in Proc. VTC'94, Stockholm, Sweden, 1994, pp. 1843-1847.

[21] E. Dahlman, B. Gudmundson, M. Nilsson, and J. Skold, "UMTS and IMT-2000 based on wideband CDMA," IEEE Commun. Mag., vol. 36, pp. 70-80, Sept. 1998. 
[22] Y. Li and N. R. Sollenberger, "Interference suppression in OFDM systems using adaptive antenna arrays," in Globecom 98. Sydney, Australia: IEEE, Nov. 8-12, 1998, vol. 1, pp. 213-218.

[23] F. W. Vook and K. L. Baum, "Adaptive antennas for OFDM," in Vehicular Technology Conf. . Ottawa, ON, Canada: IEEE, May 18-21, 1998, vol. 2, pp. 606-610.

[24] A. Czylwik, "Adaptive OFDM for wideband radio channels," in Proc. Globecom. London, U.K.: IEEE, 1996, pp. 713-718.

[25] R. F. H. Fischer and J. B. Huber, "A new loading algorithm for discrete multitone transmission," in Proc. Globecom. London, U.K.: IEEE, 1996.

[26] P. S. Chow, J. M. Cioffi, and J. A. C. Bingham, "A practical discrete multitone transceiver loading algorithm for data transmission over spectrally shaped channels," IEEE Trans. Commun., vol. 48, pp. 772-775, 1995.

[27] I. Kalet, "The multitone channel," IEEE Tran. Commun., vol. 37, pp. 119-124, Feb. 1989.

[28] H. Rohling and R. Grüheid, "Peformance of an OFDM-TDMA mobile communication system," in Proc. Globecom. London, U.K.: IEEE, 1996, pp. 1589-1593.

[29] H. Schmidt and K.-D. Kammeyer, "Adaptive Subträgerselektion zur Reduktion des Crestfaktors bei OFDM," in 3rd OFDM Fachgespräch in Braunschweig, 1998

[30] S. J. Shepherd, P. W. J. van Eetvelt, C. W. Wyatt-Millington, and S. K. Barton, "Simple coding scheme to reduce peak factor in QPSK multicarrier modulation," Electron. Lett., vol. 31, pp. 1131-1132, July 1995.

[31] A. E. Jones, T. A. Wilkinson, and S. K. Barton, "Block coding scheme for reductioon of peak to mean envelope power ratio of multicarrier transmission schemes," Electron. Lett., vol. 30, pp. 2098-2099, 1994.

[32] R. Grünheid and H. Rohling, "Ein OFDM-FDMA-Konzept für den Uplink eines Kommunikationssystemes," in 3rd OFDM Fachgespräch in Braunschweig, 1998

[33] R. W. Chang, "Synthesis of band-limited orthogonal signals for multichannel data transmission," Bell Syst. Tech. J., vol. 46, pp. 1775-1796, Dec. 1966.

[34] J. K. Cavers, "An analysis of pilot symbol assisted modulation for Rayleigh fading channels," IEEE Trans. Veh. Technol., vol. 40, no. 4, pp. 686-693, 1991 .

[35] J. M. Torrance, "Adaptive full response digital modulation for wireless communication environments," Ph.D. dissertation, Univ. of Southampton, Southampton, U.K., Mar. 1997.

[36] J. Torrance and L. Hanzo, "Optimization of switching levels for adaptive modulation in slow Rayleigh fading," Electron. Lett., vol. 32, pp. 1167-1169, June 20th, 1996.
[37] C. Berrou, A. Glavieux, and P. Thitimajshima, "Near Shannon limit error-correcting coding and decoding: Turbo-codes," in Proc. ICC, 1993, pp. 1064-1070.

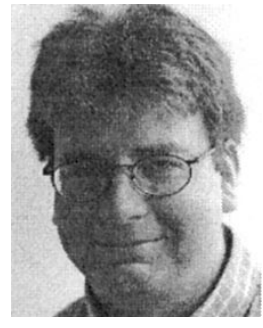

Thomas Keller studied electrical engineering at the University of Karlsruhe, Ecole Superieure d'Ingenieurs en Electronique et Electrotechnique, Paris, and the University of Southampton, Southampton, U.K., and received the Dipl.-Ing. degree in 1995. Between 1995 and 1999, he was with the Wireless Multimedia Communications Group at the University of Southampton, and received the Ph.D. degree in mobile communications from that university.

His current areas of interest include adaptive OFDM transmission, wideband channel estimation, and error correction coding. He recently joined Ubinetics, Cambridge, U.K.

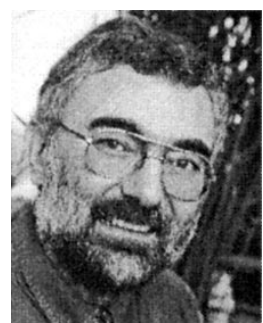

Lajos Hanzo received the Dipl. Ing. degree in electronics in 1976 and the Ph.D. degree 1983, both from the Technical University of Budapest, Hungary.

During his 24-year career in telecommunications he has held various research and academic posts in Hungary, Germany, and the U.K. Since 1986, he has been with the Department of Electronics and Computer Science, University of Southampton, U.K., and has been a Consultant to Multiple Access Communications Ltd., U.K. Currently, he holds the chair in Telecommunications. He coauthored five books on mobile radio communications, published over 300 research papers, organized and chaired conference sessions, presented overview lectures and was awarded a number of distinctions. Currently, he is managing a research team working on a range of research projects in the field of wireless multimedia communications under the auspices of the Engineering and Physical Sciences Research Council (EPSRC) U.K, the European IST Program and the Mobile Virtual Center of Excellence (VCE). He also provides a range industrial training courses. 